\title{
A MULTI-LEAD VACUUM-SEALED CAPACITIVE PRESSURE SENSOR
}

\author{
Abhijeet V. Chavan and Kensall D. Wise \\ Center for Integrated Sensors and Circuits \\ Department of Electrical Engineering and Computer Science \\ The University of Michigan, Ann Arbor, MI 48109-2122
}

\begin{abstract}
To improve the sensitivity, dynamic range and resolution of capacitive pressure sensors, it is necessary to eliminate the effects of environmental factors such as trapped gas and material thermal mismatches in addition to reducing parasitics. If the desired sensor signal can be amplified in the sealed transducer cavity itself, then the effects of externally-induced noise will be eliminated almost entirely. This work reports a new multi-lead absolute capacitive pressure sensor which lends itself to achieving these goals. The sensor has a resolution of $25 \mathrm{mT}$ Torr over a pressure range from 500 to 800 Torr with a TCO at 750 Torr of $<1000 \mathrm{ppm} /{ }^{\circ} \mathrm{C}$ and a TCS of $1350 \mathrm{ppm} / \mathrm{C}$. The parasitic capacitance on individual leads is $500 \mathrm{fF}$, which is an order of magnitude lower than a previouslyreported vacuum-sealed device [1]. The new device structure also eliminates the need to protect the outer silicon body when operating in high humidity. The resistances of the lead transfers accessing the sealed cavity are of the order of 50 ohms. The device is completely batch fabricated and provides a general purpose MEMS process for future circuit integration within sealed cavities where moving micromechanical structures are enclosed.
\end{abstract}

\section{INTRODUCTION}

Silicon-based capacitive pressure sensors are increasingly being used in a variety of applications to achieve high resolution and low temperature sensitivity. As compared to piezoresistive pressure sensors, capacitive pressure sensors also have greatly reduced power consumption. Capacitive devices in general are more susceptible to noise coupling from the environment, however, which requires the readout circuit to be in close proximity to the actual transducer. The development of a lead transfer process having low parasitics between the vacuum-sealed cavity and the outside world is a challenging problem. The sensor described here is a second-generation multi-transducer device suitable for use over the barometric pressure range from 500 to 800 Torr with a resolution of $25 \mathrm{mT}$ Torr. This high resolution is maintained in the face of atmospheric offset pressure over a temperature range from $25^{\circ} \mathrm{C}$ to $85^{\circ} \mathrm{C}$. Parasitic capacitance in parallel with the sensor capacitance has a different thermal behavior than the actual sensing element and thus complicates temperature compensation. This device significantly reduces such parasitics and eliminates any leakage paths which may occur along the leads.

\section{STRUCTURAL DESIGN AND OPERATION}

A new device structure with multiple stacked polysilicon and dielectric layers is employed. Each transducer can have several lead transfers from the internal reference cavity to the outside world and provides additional Ti/Pt electrodes to block the electric field across the entire cavity and getter any outdiffusing oxygen ions. To achieve this, two levels of polysilicon are used. Polysilicon-glass seals are known to be hermetic [2]. The sensor cross-section is diagrammed in Fig. 1 and consists of vacuumsealed capacitors realized in a silicon-on-glass dissolved-wafer process. In the structure shown in Fig. 1, the polysilicon-1 layer near the glass forms a continuous sealing ring while the polysilicon-2 layer forms a bridge for the individual lead transfers. Using this approach, wafer-level bonds can be made to 7740 glass. As is evident, the need for glass drilling, epoxy seals, or special metal seals $[3,4]$ is eliminated. In addition since the vacuum seal is done at wafer level before etch-back, the cavity is never exposed to the etch, and stiction problems are avoided. A significant practical advantage is that a metal or polysilicon electrode which is on the $\mathrm{p}++$ silicon diaphragm inside the cavity, but is isolated from it electrically, can be transferred outside of the cavity. This eliminates the need to passivate the exposed $\mathrm{p}++$ silicon surface in case of operation under moist and dirty conditions because the $\mathrm{p}++$ housing forms only the physical transducer but is electrically isolated from the electrode. Fig. 1(c) shows a cross-section of this scheme.

The overall pressure range is divided among different transducers having diaphragm diameters ranging from 920 to $1100 \mu \mathrm{m}$ with a gap separation of $9.8 \mu \mathrm{m}$ and a diaphragm thickness of $2.4 \mu \mathrm{m}$. The diaphragm has a centrally stiffened area to obtain first-order linearization of the output characteristics. One of the transducers is used for coarse pressure sub-range selection and then the appropriate segment tranducers is read-out for obtaining higher resolution. The high atmospheric offset keeps the diaphragms heavily deflected and results in an operational gap between 0.8 and $0.2 \mu \mathrm{m}$, which in turn provides high sensitivity for the device. The device is designed to operate with a resultant diaphragm tensile stress of $\sim 25 \mathrm{Mpa}$. In Fig. la, the internal sensing lead on the glass is transferred to polyl and then through a contact opening to poly 2 , back again to polyl, and then back to metal externally using Pt-Pt compression bonds formed during the electrostatic bonding operation. Since the potential of the polysilicon must be connected to the silicon bulk during bonding, but must be well isolated later, lateral polysilicon tabs are used to contact the lightly-doped portion of the bulk. This area is automatically removed during the etch-back process to provide the required isolation. Permanent contacts to the top silicon electrode are made via similar transfers to the $\mathrm{p}+$ body region as shown in Fig. lb. In the case of a multi-lead device, the majority of the anchor formed by the polysilicon ring is electrically isolated from the leads themselves. Thus, it is important to be able to do probe (batch) testing to verify this isolation. Special contacts are provided to the rim to test for this isolation. Figure 2(a) shows a complete five transducer sensor with an on-chip reference capacitor in the center of the lower row. This pressure- 
independent reference capacitor tracks the pressure dependent transducers over temperature and is used differentially with a switched-capacitor readout circuit. Fig. 2(b) views a single transducer, showing all lead transfers as seen through the glass.

(a)

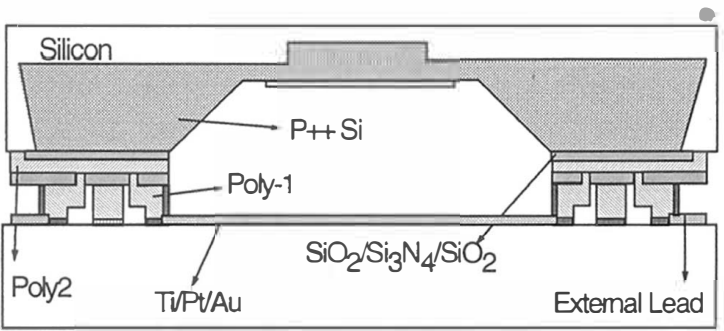

(b)

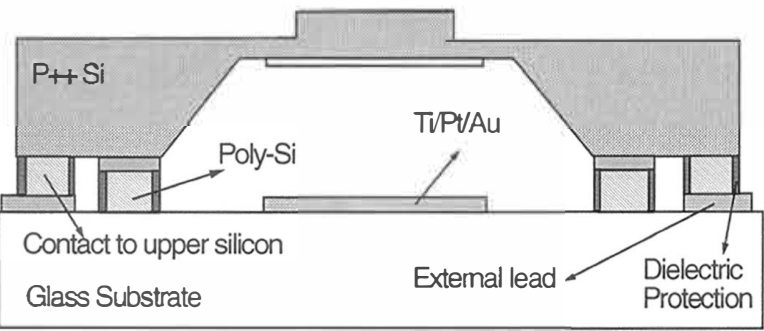

(c)

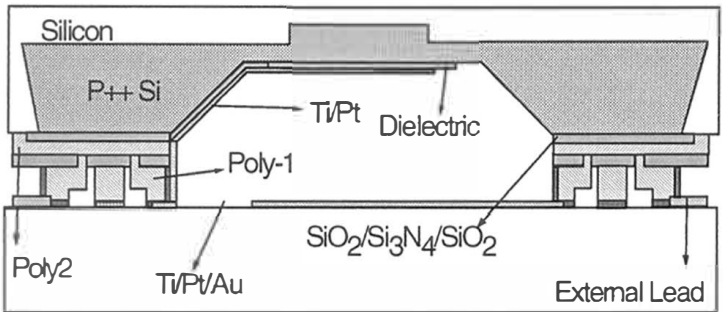

Fig. 1: Cross-sections of the capacitive sensor. In (a) the cut is shown through the lead transfer that brings the glass electrode out of the cavity. A tab used to contact the wafer bulk during bonding is also shown. In (b) the cut is shown along a device diagonal. The inner ring forms the vacuum seal; the outer ring provides a permanent contact to the silicon electrode. (c) Scheme showing the lead transfers with both electrodes isolated from the silicon bulk.

\section{FABRICATION PROCESS}

A compressed process flow is shown in Fig. 3. The device is fabricated using a ten-mask bulk-micromachined dissolved wafer process. As shown in Fig. 3(a), the silicon process starts with a $\mathrm{KOH}$ recess etch of about $7 \mu \mathrm{m}$. Subsequently deposited layers can compensate for any variation in this etch depth. A patterned masking oxide (Fig $3 \mathrm{~b}$ ) is used to define the anchor area using a solid-source deep boron diffusion at $1175^{\circ} \mathrm{C}$. A nominal etch-stop depth of $15 \mu \mathrm{m}$ is obtained. This is followed by a shallow boron diffusion step to define the diaphragm, which is approximately $2.4 \mu \mathrm{m}$ thick. The next step involves the deposition of an LPCVD $\mathrm{SiO}_{2} / \mathrm{Si}_{3} \mathrm{~N}_{4} / \mathrm{SiO}_{2}$ layer having a total thickness of $0.75 \mu \mathrm{m}$. A short boron diffusion at $1000^{\circ} \mathrm{C}$ is done to reduce the resistance of the polysilicon. In addition, $\mathrm{p}+$ doping reduces the rate of attack from EDP if the protective dielectric coating on the polysilicon is broken. It is adequate to dope the polysilicon at low temperatures due to the ease of boron diffusion through the grain boundaries. The polysilicon is patterned using $\mathrm{SF}_{6}$ plasma etching. This etch step is done using a two-step lithography. In the first step, the individual leads are patterned. This is done on the polysilicon which is on top of the anchor area. The isolation formed must have straight sidewalls because of the subsequent isolation refill using $\mathrm{SiO}_{2} / \mathrm{Si}_{3} \mathrm{~N}_{4} / \mathrm{SiO}_{2}$. In the second etch step, the polysilicon from the recesses is etched. The need for two steps is due to the substantial difference in height for patterns on the anchor and patterns in the recess. After etching, the polysilicon and the dielectric layer on the diaphragm area are removed using wet etching. This is followed by a second LPCVD $\mathrm{SiO}_{2} / \mathrm{Si}_{3} \mathrm{~N}_{4} / \mathrm{SiO}_{2}$ layer with a total thickness of $0.75 \mu \mathrm{m}$. Contact openings are made for the subsequent polysilicon-1 layer to make contact to the: a) silicon bulk along the periphery to form the tabs useful in bonding, and b) along the leads to form the electrical bridges for lead transfer. This is followed by the deposition of a $1.2 \mu \mathrm{m}$-thick layer of polysilicon-1, which is p++ doped at $1000^{\circ} \mathrm{C}$. A CMP (chemical mechanical polishing) step is used to achieve a surface roughness of $<500 \AA$-rms and to overcome any non-planarities due to wafer bowing [7]. For polishing polysilicon, it has been observed that
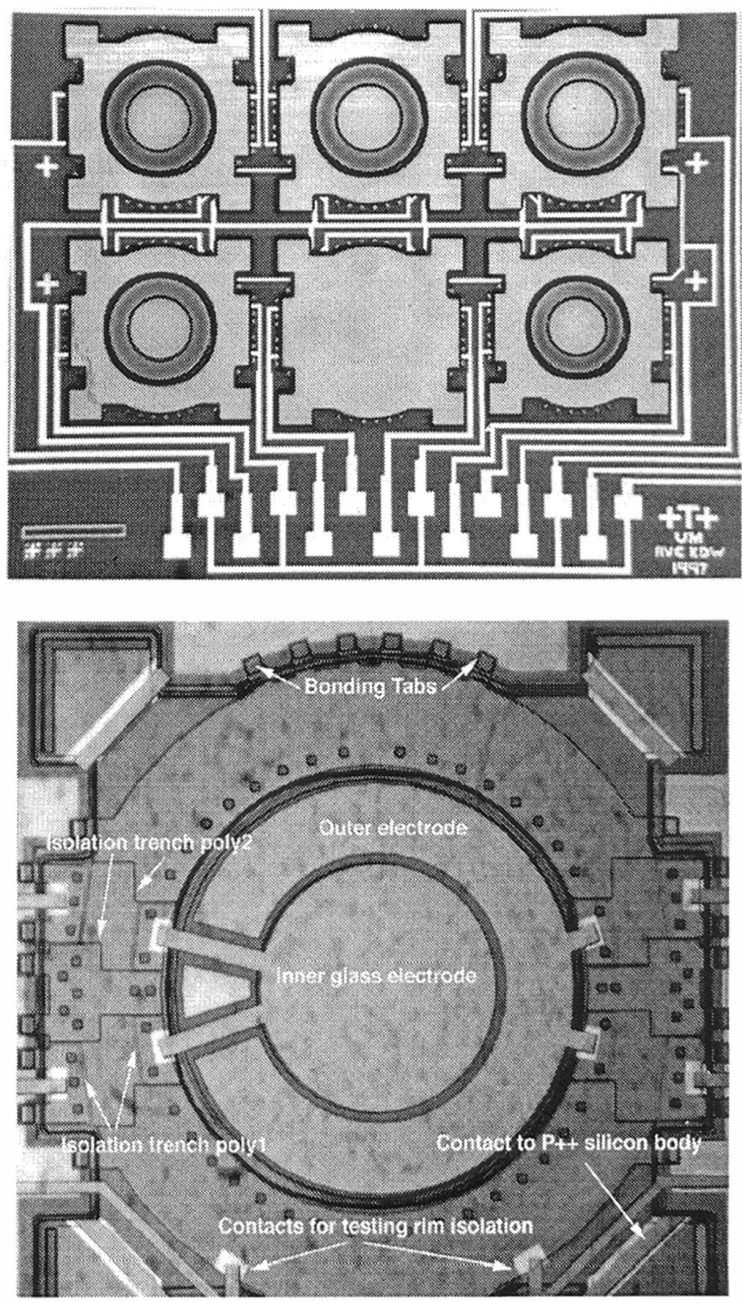

Fig. 2: Top views of the fabricated devices. In (a) five transducers are formed per die having staggered diameters and pressure ranges. A reference capacitor can be seen in the center of the lower row. In (b) the view is through the glass side of a completed transducer. 
colloidal silica-based slurries give a good surface finish devoid of any scratches and other CMP related defects [5]. It is important to immediately clean the wafers after CMP to avoid any residues. which are difficult to remove once the wafers are dry This is followed by another $\mathrm{Si}_{3} \mathrm{~N}_{4}$ layer of $800 \AA$ thickness, which provides protection to the polysilicon from attack by EDP. Another thin dielectric layer is now deposited on the diaphragm area to provide isolation for the two electrodes of the capacitor and provide stress compensation. The dielectric layer on the anchor areas is patterned so as to cover only the anchor area and permit contact openings. Ti-Pt is evaporated so as to contact the polysilicon and produce a total height of about $500 \AA$. At this point, the polysilicon is completely covered by a dielectric layer and has two metal contact areas. Due to the loss of some polysilicon during boron diffusion and CMP, the total height of the dielectric layer and polysilicon stack is about $2.8 \mu \mathrm{m}$. Thus, we have a total recess depth of $9.8 \mu \mathrm{m}$. This forms the working gap distance for the capacitor.

The glass processing consists of depositing approximately $250 / 350 / 1000 \AA$ of a composite Ti-Pt-Au layer. The Au is then etched back in the contact areas. The glass is partially diced at this point so that the devices can be easily separated after the wafer dissolution step. After this step, we perform wafer-level anodic bonding to 7740 glass in vacuum ( $1 \times 10^{-6}$ Torr); the structure is as shown in Fig. 3(g). Due to poor heat transfer in vacuum, it is important to first heat the wafers to $400^{\circ} \mathrm{C}$ in rough vacuum and then pump down the bonding chamber to higher vacuum levels. Also, pre-heating the wafers for 30 minutes helps out-diffusion of gases from the inner-walls of the sealed cavity, which is subsequently evacuated via the recessed areas between cavities when high vacuum is applied. While trying to bond wafers which have disjointed bond surfaces, a continuous bond front which can pull in the non-uniform parts of a wafer cannot be formed, and it is imperative that the surface flatness of the silicon wafer be uniform to obtain a high yield. Use of a graphite disk which covers the entire glass surface and an electrode pressure of at least $2500 \mathrm{mbar}$ also contribute significantly to a better bond yield. The next step is to dissolve the wafer in EDP to obtain the final structure as shown in Fig. 3(h). As can be seen, the diaphragm is heavily deflected under normal atmospheric conditions.

\section{TEST RESULTS}

The device was characterized for both dynamic behavior and DC parameters such as lead resistance and parasitic capacitance. The device was also tested after interfacing it to a switchedcapacitor readout circuit. Calibration/compensation is done in software to achieve the resolution of $25 \mathrm{mT}$ Torr. Each transducer has two redundant parallel lead transfers for each electrode with a nominal resistance of about $50 \mathrm{ohms}$ and a TCO of $1000 \mathrm{ppm} /{ }^{\circ} \mathrm{C}$. As mentioned earlier, the high parasitic capacitance observed with the first-generation device has been reduced to 500fF, which is about $5 \%$ of the nominal sensor capacitance. The parasitic capacitance is primarily dependent on the width of the rim, the thickness of the dielectric layer between the p++ silicon and the polysilicon, and the width of the polysilicon bridge formed in the poly-2 layer. An additional ring of $\mathrm{Ti} / \mathrm{Pt}$ metal is used on the glass to reduce the open glass surface exposed to the cavity. The $\mathrm{Ti}$ is effective in gettering any $\mathrm{O}_{2}$ ions outdiffusing from the glass. The gas pressure in the cavity has been characterized earlier [1] to be in the $200 \mathrm{mT}$ Torr range. The additional Ti/Pt ring as seen in Fig. 2(b) along with the main metal electrode on glass effectively blocks the high electric field during bonding from the entire cavity.
This is a necessary feature if future circuitry is to be placed inside the cavity $[8,9,10]$. The multi-lead structure also makes it feasible to have an independent metal electrode on the silicon isolated from the silicon body, thereby eliminating the need to protect the etched back silicon bulk during operation in humid environments. Fig. 4(a) shows the behavior of a typical device.

(a)

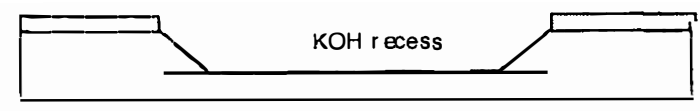

(b)
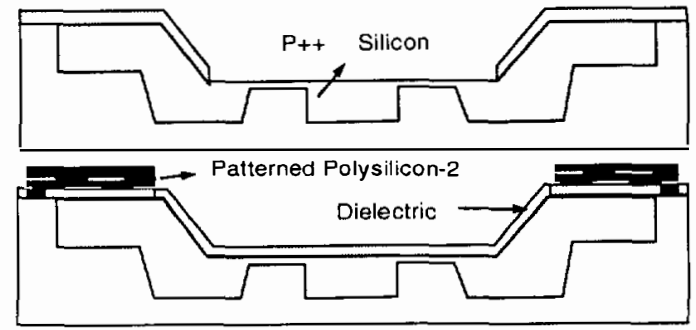

(d)

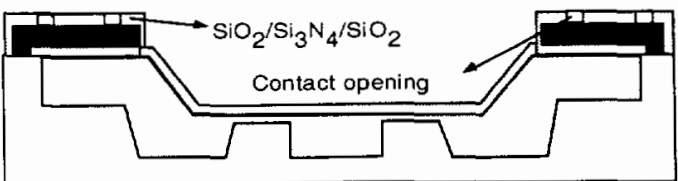

(e)

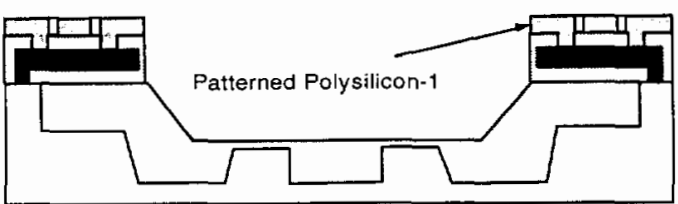

(f)

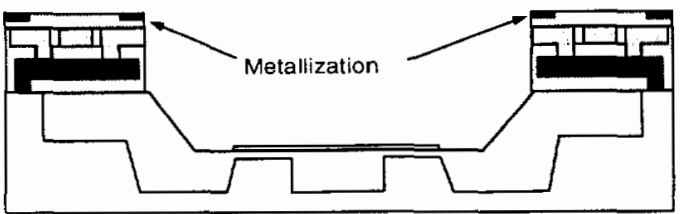

(g)

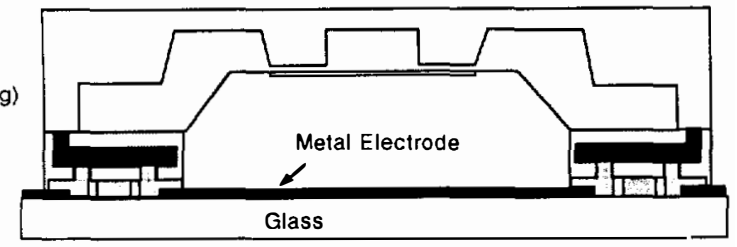

(h)

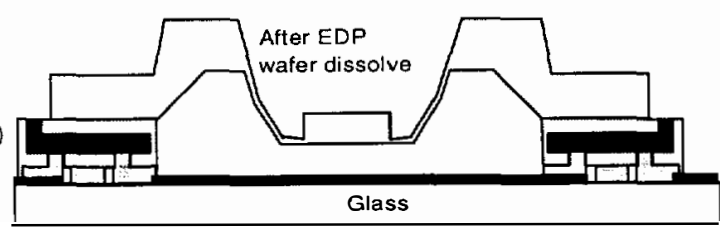

Fig. 3: Process flow for the multi-lead vacuum-sealed capacitive pressure sensor.

The nominal sensitivity for the segment devices is $24 \mathrm{fF} / \mathrm{Torr}$ (2450 ppm/Torr). The TCO at 750 Torr is $1350 \mathrm{ppm} /{ }^{\circ} \mathrm{C}$ and is primarily due to the mismatch of expansion coefficients of the anchor materials and the glass. The TCS is about $1000 \mathrm{ppm} /{ }^{\circ} \mathrm{C}$. The resolution of $25 \mathrm{mT}$ Torr is achieved after interfacing to a programmable gain switched-capacitor readout circuit and doing digital compensation in software. In the case of this sensor, each transducer in the device has a range of about 50 Torr. The maximum pressure sensitivity after interfacing to a switchedcapacitor circuit, while still covering the entire 50 Torr range, is 
$0.06 \mathrm{mV} / \mathrm{mT}$ Torr. In a $5 \mathrm{~V}$ system, $25 \mathrm{mT}$ Torr resolution is obtained using a $12 \mathrm{~b} A / D$ after allowing some margin for noise. In this case, since we have software-programmable gain, we can use different transducers with higher gain to achieve a resolution of 25 mTorr.
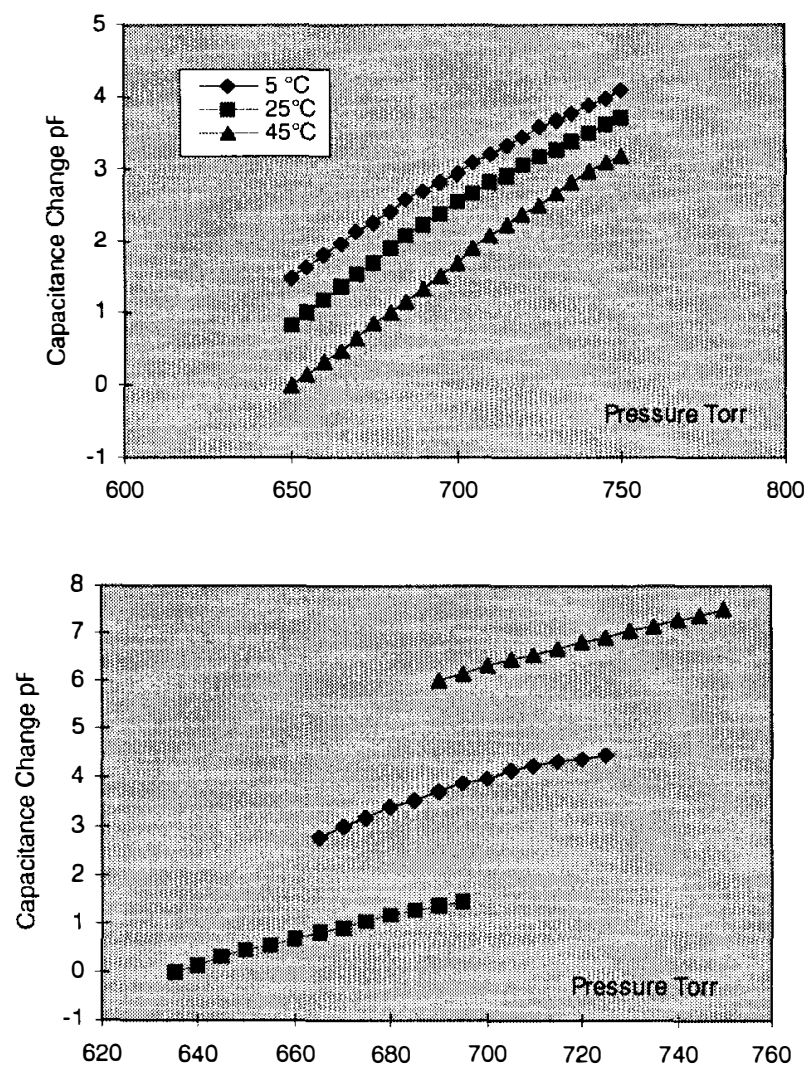

Fig. 4: (a) Typical pressure response for a single transducer over temperature. (b) Response of three different segment transducers.

In order to evaluate the long-term stability of the highly deflected boron-diffused membranes and the integrity of the polysilicon vacuum seals, we have been monitoring a number of of devices for over a year. The total observed variation in sensitivity of the global transducer was less than $\pm 30 \mathrm{ppm} / \mathrm{mmHg}$ for a baseline sensitivity of $1050 \mathrm{ppm} / \mathrm{mmHg}$. At room temperature, the variations in $\mathrm{C}_{0}$ (the $760 \mathrm{Torr}, 25^{\circ} \mathrm{C}$ baseline capacitance) for the global transducer were less than $\pm 12 \mathrm{fF}$ for a baseline $\mathrm{C}_{0}$ of $12.050 \mathrm{pF}$, measured using a HP4284A LCR meter. Some of these changes are likely due to present measurement accuracy. We see no significant unidirectional shift in baseline capacitance or in the sensitivity of these membranes. Long-term detailed testing of the devices is continuing using DH Instruments PPC2 calibrator.

\section{CONCLUSIONS}

A vacuum-sealed multi-lead/multi-transducer capacitive barometric pressure sensor employing a robust batch process has been fabricated. Two polysilicon layers are used to achieve lead transfers from the inside of the cavity and form a continuous sealing ring. A completely-isolated metal electrode on the silicon side can be used to eliminate need for passivation of the dissolved bulk silicon. Elimination of stiction problems, higher bandwidth capability, and adaptability to other glass-Si MEMS devices make this a suitable process for a variety of integrated MEMS applications. By operating at a gap spacing of between $0.3 \mu \mathrm{m}$ and $0.8 \mu \mathrm{m}$, the sensor achieves a resolution of $25 \mathrm{mTorr}$ under atmospheric offset pressures and has been stable for over one year.

\section{ACKNOWLEDGMENTS}

The authors would like to express their thanks to Mr. Larry Jordan of Delphi Delco Electronics Corporation and Jeff Kempinsky of the GM Research Laboratories for their assistance with the anodic bonding of the wafers. We would also like to thank Chuck Geyer of Logitech, Inc., for assistance with the CMP process. This project is supported by DARPA contract DABT6395-C-0111. Abhi Chavan is supported by a fellowship from the Delphi Delco Electronics Corporation.

\section{REFERENCES}

[1]. A. V. Chavan and K. D. Wise, "A Batch-Processed VacuumSealed Capacitive Pressure Sensor," Digest IEEE Int. Conf. on Solid-State Sensors and Actuators (Transducers '97), Chicago, pp. 1449-1452, June 1997.

[2]. J. Von Arx, B. Ziaie, M. Dokmeci, and K. Najafi, "Hermeticity Testing of Glass-Silicon Packages with On-Chip Feedthroughs," Digest Int. Conf. on Solid-State Sensors and Actuators, Stockholm, pp. 244-247, June 1995.

[3]. J. M. Giachino, et al., US Patents 4261086 (4/1981) and 4386453 (6/1983) and Peters, et al., US Patent 4586109 (4/1986).

[4]. M. Esashi, Y. Matsumoto and S. Shoji, " Absolute Pressure Sensors by Air-Tight Electrical Feedthrough Structure", Sensors and Actuators, A21-A23, 1990, pp. 1048-1052.

[5]. DeJule Ruth, "CMP Challenges below a Quarter Micron," Semiconductor International, November 1997, pp 55-60.

[6]. B. Puers, E. Peeters, A. Van Den Bossche and W. Sansen, "A Capacitive Pressure Sensor with Low Impedance Output and Active Suppression of Parasitic Effects", Sensors and Actuators, A21-A23, 1990 pp 108-114.

[7]. J. Sniegowski, "Chemical Mechanical Polishing: Enhancing the Manufacturability of MEMS," SPIE Vol. 2879, 1996, pp. 104 115 .

[8]. C. Sander, J. Knutti and J. Meindl, "A Monolithic Capacitive Pressure Sensor with Pulse-Period Output," IEEE Tran on Electron Devices, Vol. ED-27, No. 5 May 1980, pp 927-930.

[9] Y. Gianchandani, K. J. Ma and K. Najafi, "A CMOS Dissolved-Wafer Process for Integrated $\mathrm{p}++$ Microelectromechanical Systems", Digest IEEE Int Conf on Solid-State Sensors and Actuators, (Stockholm) pp. 79-82, June 1995.

[10]. T. Kudoh, Shuichi Shoji and Masayoshi Esashi, "An Integrated miniature Capacitive Pressure Sensor,"Sensors and Actuators, A-29, 1991, pp 185-193. 